\title{
Fast, accurate enthalpy differences in spin crossover crystals from DFT $+\mathrm{U}$
}

\author{
Miriam Ohlrich* and Ben J. Powell* \\ School of Mathematics and Physics, University of Queensland, Brisbane \\ E-mail: miriam.ohlrich@uq.net.au; powell@physics.uq.edu.au
}

\begin{abstract}
Spin crossover materials are bi-stable systems with potential applications as molecular scale electronic switches, actuators, thermometers, barometers and displays. However, calculating the enthalpy difference, $\Delta H$, between the high spin (HS) and low spin (LS) states has been plagued with difficulties. For example, many common density functional theory (DFT) methods fail to even predict the correct sign of $\Delta H$, which determines the low temperature state. Here, we study a collection of $\mathrm{Fe}(\mathrm{II})$ and $\mathrm{Fe}(\mathrm{III})$ materials, where $\Delta H$ has been measured, and which has previously been used to benchmark density functionals. The best performing hybrid functional, TPSSh, achieves a mean absolute error compared to experiment of $11 \mathrm{~kJ} \mathrm{~mol}^{-1}$ for this set of materials. However, hybrid functionals scale badly in the solid state; therefore, local functionals are preferable for studying crystalline materials, where the most interesting SCO phenomena occur. We show that both the Liechtenstein and Dudarev DFT $+\mathrm{U}$ methods are a little more accurate than TPSSh. The Dudarev method yields a mean absolute error of $8 \mathrm{~kJ} \mathrm{~mol}^{-1}$ for $U_{\text {eff }}=1.6 \mathrm{eV}$. However, the MAE for both TPSSh and DFT $+\mathrm{U}$ are dominated by a single material - if this is excluded from the set then DFT $+\mathrm{U}$ achieves chemical accuracy. Thus, DFT $+\mathrm{U}$ is an attractive option for calculating the proper-
\end{abstract}


ties of spin crossover crystals, as its accuracy is comparable to that of meta-hybrid functionals, but at a much lower computational cost.

\section{Introduction}

Spin crossover (SCO) is a phenomenon where the equilibrium state of a material can transition between a high spin (HS) and low spin (LS) state with changes in temperature, pressure, applied magnetic fields or light irradiation. ${ }^{1}$ Many SCO materials are pseudo-octahedral coordination complexes of first row transition metal ions with open d-orbitals $\left(d^{4}-d^{7}\right){ }^{2}$ The HS state occurs when the crystal field splitting, $\Delta_{\mathrm{cf}}$, is smaller than the d-orbital electron pairing energy and the LS state occurs in the opposite case. ${ }^{3}$ In SCO materials the enthalpy difference between the HS and LS states, $\Delta H=H_{\mathrm{HS}}-H_{\mathrm{LS}}$, is typically less than $50 \mathrm{~kJ} \mathrm{~mol}^{-1} .^{4}$ As SCO often occurs in large coordination complexes and coordination polymers, this is challenging for first principles approaches to accurately and reliably capture.

Density functional theories (DFT) utilizing hybrid functionals, which include some exact exchange can predict $\Delta H$ accurately enough to allow the prediction of many material properties. Notably, Jensen and Cirera ${ }^{5}$ reported that the meta-hybrid TPSSh, with $10 \%$ exact exchange, gave a mean absolute error relative to experiment (MAE) of $11 \mathrm{~kJ} \mathrm{~mol}^{-1}$ for $\Delta H$ for a range of Fe based SCO complexes. However, many of the most interesting properties of SCO materials, from both the fundamental ${ }^{6-15}$ and applications ${ }^{15-24}$ perspectives, result from the interplay between the changes in enthalpy and entropy when a single molecule changes spin-state, and frustrated elastic interactions between complexes in the solid state. The elastic interactions are fundamentally a property of the solid state, rather than a single complex. However, in the solid state, the exact exchange component of hybrid functionals becomes prohibitively time consuming. This has motivated several groups to investigate whether similar or higher accuracy results can be achieved without the use of hybrid density functionals. ${ }^{25-31}$ 
Pure density functionals, such as the local density approximation (LDA) and generalized gradient approximations (GGA), tend to over-delocalize the valence electrons. This is because these approximations for the exchange correlation functional do not fully cancel out the self-interaction term in the Hartree potential. Thus, there is some residual repulsion between each electron and itself in the model, which forces the electrons further away from the nucleus. ${ }^{32}$ This is why DFT calculations based on pure functionals fail to accurately predict the properties of materials involving transition metals with open $d$ or $f$ orbitals: where the valence electrons are strongly interacting and localized. ${ }^{33,34}$

One way to counter this over-delocalisation is to use an LDA or GGA functional with an added Hubbard model-like potential term (the DFT $+\mathrm{U}$ method). ${ }^{32,35-37}$ This potential term includes electrostatic interactions between two electrons in the same orbital and the energy associated with the exchange of electrons between orbitals on the same atom. The inclusion of this on-site Coulomb interaction $(U)$, and the electron exchange interaction $(J)$, increases the electron localization: approximately correcting the over-delocalization of valence electrons in DFT. ${ }^{32,35}$

Since DFT $+\mathrm{U}$ uses a pure density functional for all but the strongly correlated $d$ orbital electrons, and the added potential terms only act locally, its computational time is comparable to pure functionals like LDA and GGA, even in the solid state. In contrast, the computational times for hybrid functionals dramatically increase with the size of the crystal. $^{32}$

Most previous papers that have discussed the use of $\mathrm{DFT}+\mathrm{U}$ for spin crossover materials have tuned the $U$ and $J$ parameters to reproduce the properties of a single material. ${ }^{25-30}$ Vela et al. ${ }^{31}$ is the only systematic study of the DFT $+\mathrm{U}$ parameterization we are aware of. They included an empirical treatment of the vibronic contributions and, to implement this, they needed to adjust the calculated single molecule frequencies to match the measured vibrational entropy. If such measurements are not available for molecular crystals then this approach is not possible. It is also not clear how to extend this method to frameworks and coordination 
polymers, where many important SCO phenomena are observed. ${ }^{7-10,24}$ We are, therefore, motivated to ask: can a DFT $+\mathrm{U}$ method with no experimental input and a common value of $U$ and $J$ achieve results of comparable or better accuracy than those of hybrid functionals for the spin crossover enthalpy difference for a set of spin crossover materials? To answer this question, we investigated two $\mathrm{DFT}+\mathrm{U}$ methods: the Liechtenstein method, ${ }^{37}$ which treats $U$ and $J$ as separate parameters, and the Dudarev method, ${ }^{33}$ which uses only the difference between them, $U_{\text {eff }}=U-J$.

\section{Methods}

\section{Training set}

To benchmark our DFT $+\mathrm{U}$ calculations we need a collection of spin crossover materials for which the spin transition enthalpy differences have been experimentally determined. For ease of comparison, we selected the same set that Jensen and Cirera ${ }^{5}$ used to benchmark a range of functionals (Table 1, Figure 1). However, only the iron complexes were investigated, as different $U$ and $J$ values are needed to accurately describe complexes with different central ions. 
Table 1: Spin crossover materials investigated. We present their $d$ electron configuration $\left(d^{n}\right)$; the measured enthalpy differences $(\Delta H)$, the range including both the experimental error (where reported) and differences between experiments, with MAEs calculated relative to the midpoint of this range; and the reference codes for the compounds in the Cambridge Structural Database (CSD). Ligands are abbreviated as follows: acac = acetylacetonate, trien $=$ triethylenetetramine, papth $=2$-(2-pyridylamino $)-4-(2$-pyridyl $)$ thiazole, tacn $=1,4,7$-triazacyclononane, 2 -amp $=2$-aminomethylpyridine, $\mathrm{HB}(\mathrm{pz})_{3}=$ hydrotris $($ pyrazol-1yl)borate, py (bzimH) $=2$-(2'-pyridyl)benzimidazole, and tppn = tetrakis $(2$-pyridylmethyl)1,2-propanediamine.

\begin{tabular}{c|cccc} 
No. & Material & $d^{n}$ & $\Delta H(\mathrm{~kJ} / \mathrm{mol})$ & CSD \\
\hline $\mathbf{1}$ & {$\left[\mathrm{Fe}(\text { acac })_{2}\right.$ trien $]\left(\mathrm{PF}_{6}\right)$} & $d^{5}$ & $7-17^{38,39}$ & actrfe $^{40}$ \\
$\mathbf{2}$ & {$\left[\mathrm{Fe}(\text { papth })_{2}\right]\left(\mathrm{BF}_{4}\right)$} & $d^{6}$ & $16^{41}$ & colijao $^{42}$ \\
$\mathbf{3}$ & {$\left[\mathrm{Fe}(\mathrm{tacn})_{2}\right]\left(\mathrm{Cl}_{2}\right)$} & $d^{6}$ & $21-24^{43}$ & dettol $^{44}$ \\
$\mathbf{4}$ & {$\left[\mathrm{Fe}(2-\mathrm{amp})_{3}\right]\left(\mathrm{Cl}_{2}\right)$} & $d^{6}$ & $18-25^{45}$ & fepicc $^{46}$ \\
$\mathbf{5}$ & {$\left[\mathrm{Fe}\left(\mathrm{HB}(\mathrm{pz})_{3}\right)_{2}\right]$} & $d^{6}$ & $16-22^{47,48}$ & hpzbfe $^{49}$ \\
$\mathbf{6}$ & {$\left[\mathrm{Fe}(\mathrm{py}(\mathrm{bzimH}))_{3}\right]\left(2 \mathrm{ClO}_{4}\right)$} & $d^{6}$ & $20-21^{50}$ & kokfof $^{51}$ \\
$\mathbf{7}$ & {$[\mathrm{Fe}(\mathrm{tppn})]\left(2 \mathrm{ClO}_{4}\right)$} & $d^{6}$ & $25-30^{52}$ & iqiceq $^{53}$
\end{tabular}

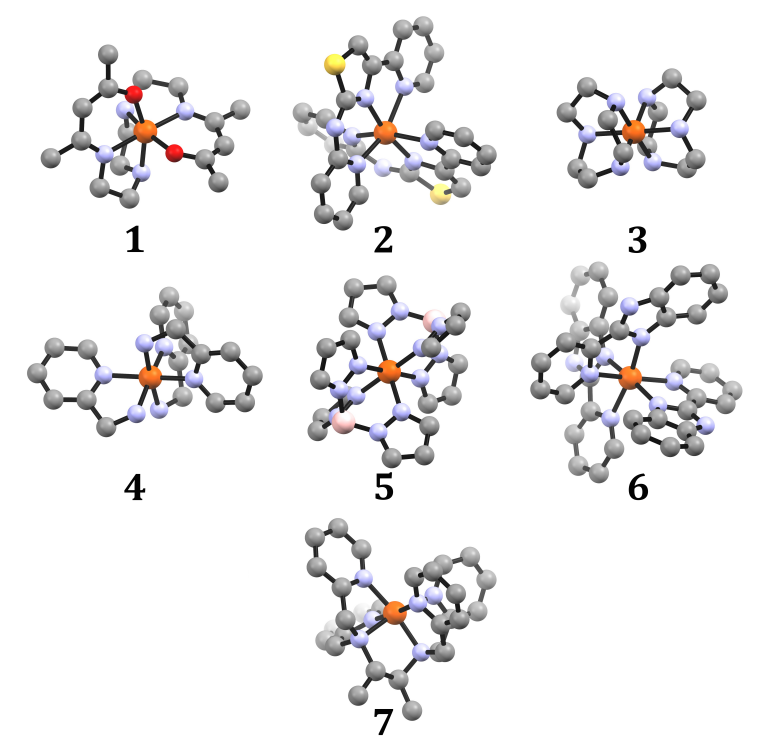

Figure 1: Structures of complexes investigated. Hydrogen atoms and counter ions are excluded for clarity.

\section{Computational details}

Where absent, hydrogen atoms were added to structures using the 'HADD' function in OLEX2. ${ }^{54}$ All DFT calculations were carried out in the Vienna Ab initio Simulation Pack- 
age (VASP). ${ }^{55-58}$ First, structural relaxations were carried out for each crystal using the PBE functional to provide both HS and LS structures. Then, DFT $+\mathrm{U}$ calculations were performed, also using the PBE functional, while gradually varying $U$ and $J$. All calculations used a plane wave basis set with a plane wave cutoff of $500 \mathrm{eV}$. The stopping condition for the minimization of the density functional was that two successive steps differed in energy by $10^{-6} \mathrm{eV}$ or less, and the Brillouin zone was sampled only at the $\Gamma$ point, because we are only interested in the total energies of the crystal. Input files and selected output for these calculations are available for download. ${ }^{59}$

\section{Results and discussion}

\section{Dudarev Approach}

$U_{\text {eff }}=U-J$ was increased in increments of $0.1 \mathrm{eV}$ from the relaxed HS and LS structures. The difference between the calculated $\Delta H$ and that measured experimentally for each material is plotted in Figure 2 .

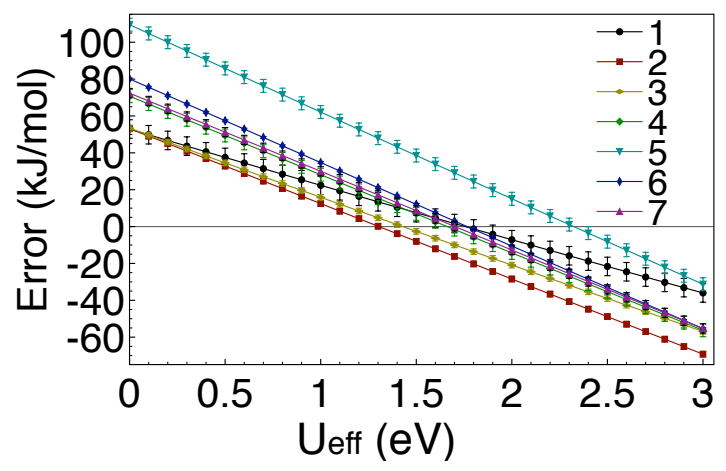

Figure 2: Error in the calculated enthalpy difference between the HS and LS states, $\Delta H$, relative to the experimentally measured values (Table 1), using the Dudarev approach with a PBE functional. The error bars represent the range of experimental values in Table 1.

Increasing $U_{\text {eff }}$ leads to a linear decrease in the spin crossover enthalpy difference for all materials. For materials 2-7, all of the gradients are very similar, whereas the gradient for material 1 (black) is clearly different. A straightforward analysis of the Hubbard (or more 
strictly Kanamori) model ${ }^{60}$ shows that different gradients should be expected for different (formal) numbers of the d electrons, in line with this finding. Orbital relaxation and hybridisation mean that such a simple calculation does not correctly predict the magnitude of the gradient. Nevertheless, it explains the linear variation of $\Delta H$ with $U_{\text {eff }}$, the very similar gradients of materials $\mathbf{2 - 7}$, and the different gradient of $\mathbf{1}$.

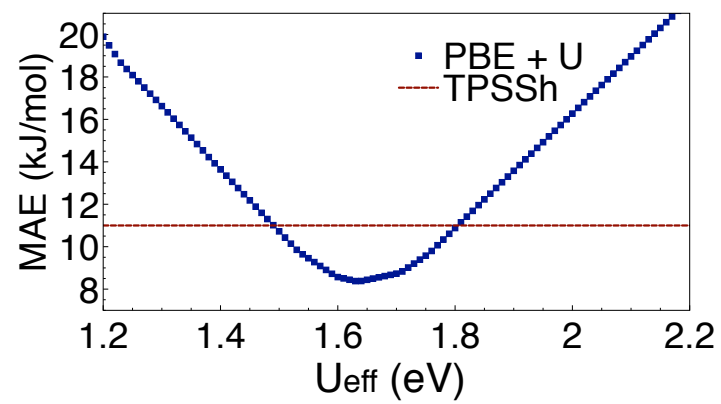

Figure 3: Mean absolute error in the spin crossover enthalpy difference for the Dudarev approach, using the PBE functional (blue squares), calculated over materials 1-7. For comparison, the MAE for the TPSSh functional for the same set of materials $\left(11 \mathrm{~kJ} \mathrm{~mol}{ }^{-1}\right.$; red line) is also shown. ${ }^{5}$ For $U_{\text {eff }}$ between 1.5 and $1.8 \mathrm{eV}$, the MAE from the DFT $+\mathrm{U}$ calculation is lower than that for the TPSSh functional. The lowest MAE $\left(8 \mathrm{~kJ} \mathrm{~mol}^{-1}\right)$ achieved for $U_{\text {eff }}=1.63 \mathrm{eV}$.

To determine the optimal value of $U_{\text {eff }}$, we calculated the mean absolute error over the entire set of materials (Figure 3). The DFT $+\mathrm{U}$ method, carried out using the Dudarev approach, marginally outperforms the TPSSh functional: the lowest MAE for the DFT $+\mathrm{U}$ method is $8 \mathrm{~kJ} \mathrm{~mol}^{-1}$, for $U_{\text {eff }}=1.63 \mathrm{eV}$, compared to a MAE of $11 \mathrm{~kJ} \mathrm{~mol}^{-1}$ for TPSSh. Note also that the minimum is extremely soft - therefore the accuracy is not strongly affected by different choices of $U_{\text {eff. }}$ The largest absolute error for any single material with this value of $U_{\text {eff }}$ is $30 \mathrm{~kJ} \mathrm{~mol}^{-1}$, which also marginally outperforms the largest absolute error for TPSSh, which was $32 \mathrm{~kJ} \mathrm{~mol}^{-1}$. The largest absolute error for both the TPSSh functional and the Dudarev approach occurs for material 5 (which is a clear outlier in Figure 2). Therefore, one may begin to suspect that the reported experimental value for this material may not be accurate. Excluding this material, the optimal value of $U_{\text {eff }}$ is $1.6 \mathrm{eV}$, with a MAE of $4.7 \mathrm{~kJ} \mathrm{~mol}^{-1}$ - chemical accuracy - and the MAE for the TPSSh functional is $7.5 \mathrm{~kJ} \mathrm{~mol}^{-1}$. 
So, the optimized value of $U_{\text {eff }}$ is only changed marginally, and the Dudarev method still yields more accurate results than the TPSSh functional.

\section{Liechtenstein Approach}

In the Liechtenstein approach, $U$ and $J$ are varied independently. The MAE over all seven materials is reported in Figure 4.

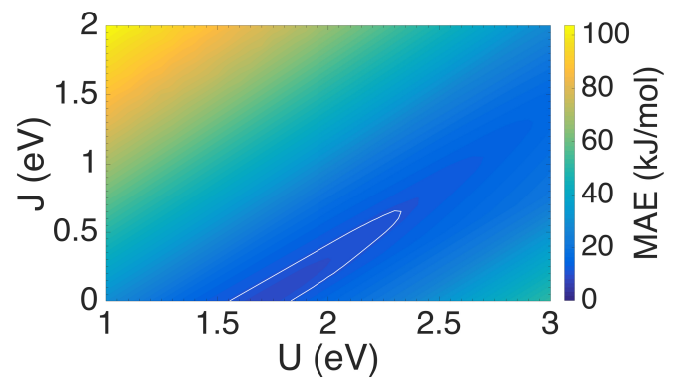

Figure 4: Mean absolute error in the spin crossover enthalpy difference for the Liechtenstein approach, using the PBE functional. These values were calculated over materials 1-7 at increments of $0.05 \mathrm{eV}$ in $U$ and $J$. The white line represents the contour with the same MAE as reported previously for the TPSSh functional, ${ }^{5}\left(11 \mathrm{~kJ} \mathrm{~mol}^{-1}\right.$ for compounds 17). All values of $U$ and $J$ within this loop give lower MAEs than the TPSSh functional, the minimum MAE being $8.7 \mathrm{~kJ} \mathrm{~mol}^{-1}$, which occurs at $U=1.7 \mathrm{eV}$, and $J=0$. This outperforms the TPSSh functional, and is very similar to the MAE found using the Dudarev method.

For $U=1.7 \mathrm{eV}$ and $J=0$ the Liechtenstein method also gives a marginally lower MAE $\left(8.8 \mathrm{~kJ} \mathrm{~mol}^{-1}\right)$ than the TPSSh functional. The largest absolute error is $29 \mathrm{~kJ} \mathrm{~mol}^{-1}$, which again occurs for material 5. Excluding this material yields a minimum MAE of $5.4 \mathrm{~kJ} \mathrm{~mol}^{-1}$ when $U=1.7 \mathrm{eV}$ and $J=0$. So, the values of $U$ and $J$ do not shift in this case, and the

results are still better than the TPSSh functional (where the MAE is $7.5 \mathrm{~kJ} \mathrm{~mol}^{-1}$ excluding material 5).

\section{Conclusion}

Both the Dudarev and Liechtenstein DFT $+\mathrm{U}$ methods give lower values for the MAE than Jensen and Cirera ${ }^{5}$ found using the TPSSh functional. However, the Dudarev method gives a 
slightly lower MAE than the Liechtenstein method, and has a lower computational time, so, based on the results for these seven materials, it is the recommended method. As we suspect that the reported experimental enthalpy difference for material $\mathbf{5}$ may not be accurate, we recommended using $U_{\text {eff }}=1.60 \mathrm{eV}$, for future calculations of the spin transition enthalpy difference in SCO materials.

The optimized values of $U_{\text {eff }}=U-J$ determined for the DFT $+\mathrm{U}$ approaches are in good agreement with previous $\mathrm{DFT}+\mathrm{U}$ calculations where $U_{\text {eff }}$ was optimized for only a single material. Values obtained for individual materials range from $U_{\text {eff }}=1.55 \mathrm{eV}$ to $2.5 \mathrm{eV} .^{25-28,30}$ Vela et al. ${ }^{31}$ found a larger value of $U_{\text {eff }}$ than us $(2.65 \mathrm{eV})$. This is reasonable as they are subtracting estimate of the vibronic contribution to $\Delta H$ made by combing experiment and theory. As $\Delta H$ monotonically decreases with $U_{\text {eff }}$ this implies that one should expect Vela $e t$ al.'s effective $U$ to be larger than ours. We also note that our approach gives a very similar accuracy to the more complicated method of Vela et al: we find a MAE of $4.7 \mathrm{~kJ} \mathrm{~mol}^{-1}$ (excluding material 5), whereas they reported a MAE of $4.3 \mathrm{~kJ} \mathrm{~mol}^{-1}$ (for a different set of materials).

Overall, using the Dudarev method with $U_{\text {eff }}=1.6 \mathrm{eV}$ is an attractive prospect. It provides a computationally inexpensive way to predict the enthalpy difference between the HS and LS states for crystal structures where no experimental input is available.

\section{Acknowledgement}

We thank Thom Aldershof, Ross McKenzie, Tanglaw Roman, Amy Thompson, and Jacob Whittaker for helpful conversations. This work was supported by the Australian Research Council through grant number DP200100305. 


\section{References}

(1) Levchenko, G. G.; Khristov, A. V.; Varyukhin, V. N. Spin crossover in iron(II)containing complex compounds under a pressure. Low Temp. Phys. 2014, 40, 571-585.

(2) Sauvage, J. P. Transition metals in supramolecular chemistry; Perspectives in supramolecular chemistry; Wiley: Chichester, England, 1999.

(3) Cotton, F. A.; Wilkinson, G.; Gaus, P. L. Basic inorganic chemistry, 3rd ed.; John Wiley: New York, 1995.

(4) Turner, J. W.; Schultz, F. A. Coupled electron-transfer and spin-exchange reactions. Coord. Chem. Rev. 2001, 219-221, 81-97.

(5) Jensen, K.; Cirera, J. Accurate computed enthalpies of spin crossover in iron and cobalt complexes. J. Phys. Chem. A 2009, 113, 10033-10039.

(6) Trzop, E.; Zhang, D.; Piñeiro Lopez, L.; Valverde-Muñoz, F. J.; Carmen Muñoz, M.; Palatinus, L.; Guerin, L.; Cailleau, H.; Real, J. A.; Collet, E. First Step Towards a Devil's Staircase in Spin-Crossover Materials. Angewandte Chemie International Edition 2016, 55, 8675-8679.

(7) Murphy, M. J.; Zenere, K. A.; Ragon, F.; Southon, P. D.; Kepert, C. J.; Neville, S. M. Guest Programmable Multistep Spin Crossover in a Porous 2-D Hofmann-Type Material. Journal of the American Chemical Society 2017, 139, 1330-1335, PMID: 28045257.

(8) Clements, J. E.; Price, J. R.; Neville, S. M.; Kepert, C. J. Hysteretic Four-Step Spin Crossover within a Three-Dimensional Porous Hofmann-like Material. Angewandte Chemie International Edition 2016, 55, 15105-15109.

(9) Halder, G. J.; Chapman, K. W.; Neville, S. M.; Moubaraki, B.; Murray, K. S.; Létard, J.-F.; Kepert, C. J. Elucidating the Mechanism of a Two-Step Spin Transition in 
a Nanoporous Metal-Organic Framework. Journal of the American Chemical Society 2008, 130, 17552-17562.

(10) Kröber, J.; Codjovi, E.; Kahn, O.; Groliére, F.; Jay, C. A spin transition system with a thermal hysteresis at room temperature. J. Am. Chem. Soc. 1993, 115, 9810-9811.

(11) Griffin, M.; Shakespeare, S.; Shepherd, H. J.; Harding, C. J.; Létard, J.-F.; Desplanches, C.; Goeta, A. E.; Howard, J. A. K.; Powell, A. K.; Mereacre, V.; Garcia, Y.; Naik, A. D.; Müller-Bunz, H.; Morgan, G. G. A Symmetry-Breaking Spin-State Transition in Iron(III). Angewandte Chemie International Edition 2011, 50, 896-900.

(12) Collet, E.; Watanabe, H.; Bréfuel, N.; Palatinus, L.; Roudaut, L.; Toupet, L.; Tanaka, K.; Tuchagues, J.-P.; Fertey, P.; Ravy, S.; Toudic, B.; Cailleau, H. Aperiodic Spin State Ordering of Bistable Molecules and Its Photoinduced Erasing. Phys. Rev. Lett. 2012, 109, 257206.

(13) Cruddas, J.; Powell, B. J. Spin-State Ice in Elastically Frustrated Spin-Crossover Materials. J. Am. Chem. Soc. 2019, 141, 19790-19799.

(14) Paez-Espejo, M.; Sy, M.; Boukheddaden, K. Elastic Frustration Causing Two-Step and Multistep Transitions in Spin-Crossover Solids: Emergence of Complex Antiferroelastic Structures. Journal of the American Chemical Society 2016, 138, 3202-3210, PMID: 26860531.

(15) Mikolasek, M. et al. Complete Set of Elastic Moduli of a Spin-Crossover Solid: SpinState Dependence and Mechanical Actuation. J. Am. Chem. Soc. 2018, $140,8970$.

(16) Bousseksou, A.; Molnár, G.; Salmon, L.; Nicolazzi, W. Molecular spin crossover phenomenon: recent achievements and prospects. Chem. Soc. Rev. 2011, 40, 3313-3335.

(17) Gütlich, P.; Goodwin, H. Spin Crossover in Transition Metal Compounds I; Topics in Current Chemistry; Springer: Berlin, 2004; Vol. 233; pp 1-47. 
(18) Halcrow, M. A. Spin-Crossover Materials: Properties and Applications; John Wiley and Sons Ltd: West Sussex, 2013.

(19) Kahn, O.; Martinez, C. J. Spin-Transition Polymers: From Molecular Materials Toward Memory Devices. Science 1998, 279, 44-48.

(20) Hauser, A. Intersystem crossing in Fe(II) coordination compounds. Coordination Chemistry Reviews 1991, 111, 275 - 290.

(21) Kahn, O.; Kröber, J.; Jay, C. Spin Transition Molecular Materials for displays and data recording. Advanced Materials 1992, 4, 718-728.

(22) Freysz, E.; Montant, S.; Létard, S.; Létard, J.-F. Single laser pulse induces spin state transition within the hysteresis loop of an Iron compound. Chemical Physics Letters 2004, 394, $318-323$.

(23) Fouché, O.; Degert, J.; Jonusauskas, G.; Daro, N.; Létard, J.-F.; Freysz, E. Mechanism for optical switching of the spin crossover [Fe(NH2-trz)3](Br)2 $3 \mathrm{H} 2 \mathrm{O}$ compound at room temperature. Phys. Chem. Chem. Phys. 2010, 12, 3044-3052.

(24) Jureschi, C.; Linares, J.; Rotaru, A.; Ritti, M.; Parlier, M.; Dîrtu, M.; Wolff, M.; Garcia, Y. Pressure Sensor via Optical Detection Based on a 1D Spin Transition Coordination Polymer. Sensors 2015, 15, 2388-2398.

(25) Lebègue, S.; Pillet, S.; Ángyán, J. G. Modeling spin-crossover compounds by periodic DFT + U approach. Phys. Rev. B 2008, 78, 024433.

(26) Bučko, T.; Hafner, J.; Lebègue, S.; Ángyán, J. G. Spin crossover transition of $\mathrm{Fe}(\text { phen })_{2}(\mathrm{NCS})_{2}$ : periodic dispersion-corrected density-functional study. PCCP $\mathbf{2 0 1 2}$, 14,5389 . 
(27) Gueddida, S.; Alouani, M. Spin crossover in a single Fe(phen $)_{2}(\mathrm{NCS})_{2}$ molecule adsorbed onto metallic substrates: An ab initio calculation. Phys. Rev. B Condens. Matter $2013,8 \%$.

(28) Gueddida, S.; Alouani, M. Calculated impact of ferromagnetic substrate on the spin crossover in a $\mathrm{Fe}(1,10 \text { - phenanthroline })_{2}(\mathrm{NCS})_{2}$ molecule. Phys. Rev. B 2016, 93, 184433.

(29) Paulsen, H. Periodic Density Functional Calculations in Order to Assess the Cooperativity of the Spin Transition in Fe(phen $)_{2}(\mathrm{NCS})_{2}$. Magnetochemistry 2016, 2.

(30) Vela, S.; Novoa, J. J.; Ribas-Arino, J. Insights into the crystal-packing effects on the spin crossover of $[\mathrm{FeII}(1-\mathrm{bpp})] 2+$-based materials. Phys. Chem. Chem. Phys. 2014, 16, $27012-27024$.

(31) Vela, S.; Fumanal, M.; Ribas-Arino, J.; Robert, V. Towards an accurate and computationally-efficient modelling of Fe(ii)-based spin crossover materials. Phys. Chem. Chem. Phys. 2015, 17, 16306-16314.

(32) Himmetoglu, B.; Floris, A.; de Gironcoli, S.; Cococcioni, M. Hubbard-Corrected DFT Energy Functionals: The LDA + U Description of Correlated Systems. Int. J. Quantum Chem. 2014, 114, 14-49.

(33) Dudarev, S.; Botton, G.; Savrasov, S.; Humphreys, C.; Sutton, A. Electron-energy-loss spectra and the structural stability of nickel oxide: An LSDA $+\mathrm{U}$ study. Phys. Rev. B 1998, 5\%, 1505-1509.

(34) Solovyev, I.; Dederichs, P.; Anisimov, V. Corrected atomic limit in the local-density approximation and the electronic structure of d impurities in rubidium. Phys. Rev. B, Condens. Matter 1994, 50, 16861-16871. 
(35) Anisimov, V.; Zaanen, J.; Andersen, O. Band theory and Mott insulators: Hubbard U instead of Stoner I. Phys. Rev. B, Condens. matter 1991, 44, 943-954.

(36) Anisimov, V.; Solovyev, I.; Korotin, D.; Czyzyk, M.; Sawatzky, G. Density-functional theory and $\mathrm{NiO}$ photoemission spectra. Phys. Rev. B, Condens. matter 1993, 48, 16929-16934.

(37) Liechtenstein, A.; Anisimov, V.; Zaanen, J. Density-Functional theory and strong interactions - orbital ordering in Mott-Hubbard insulators. Phys. Rev. B 1995, 52, R5467R5470.

(38) Binstead, R. A.; Beattie, J. K.; Dewey, T. G.; Turner, D. H. Intersystem-crossing dynamics and coordination geometry changes observed by ultrasonic and laser temperature-jump relaxation of the ${ }^{2} \mathrm{~T} \rightleftharpoons{ }^{6} \mathrm{~A}$ spin equilibrium of hexadentate iron(III) complexes in solution. J. Am. Chem. Soc. 1980, 102, 6442-6451.

(39) Dose, E. V.; Murphy, K. M. M.; Wilson, L. J. Synthesis and spin-state studies in solution of $\gamma$-substituted tris( $\beta$-diketonato) iron(III) complexes and their spin-equilibrium $\beta$ ketoimine analogues derived from triethylenetetramine. Inorg. Chem. 1976, 15, 26222630.

(40) Sinn, E.; Sim, G.; Dose, E. V.; Tweedle, M. F.; Wilson, L. J. Iron(III) chelates with hexadentate ligands from triethylenetetramine and .beta.-diketones or salicylaldehyde. Spin state dependent crystal and molecular structures of $\left[\mathrm{Fe}(\mathrm{acac}){ }_{2}\right.$ trien] $\mathrm{PF}_{6}$ $(S=5 / 2),\left[\mathrm{Fe}(\operatorname{acac} \mathrm{Cl})_{2} \operatorname{trien}_{\mathrm{PF}_{6}}(S=5 / 2),\left[\mathrm{Fe}(\mathrm{sal})_{2} \operatorname{trien}\right] \mathrm{Cl}_{2} \mathrm{H}_{2} \mathrm{O}(S=1 / 2)\right.$, and $\left[\mathrm{Fe}(\mathrm{sal})_{2}\right.$ trien $] \mathrm{NO}_{3} \mathrm{H}_{2} \mathrm{O}(S=1 / 2)$. J. Am. Chem. Soc. 1978, 100, 3375-3390.

(41) Beattie, J. K.; Binstead, R. A.; West, R. J. Intersystem crossing observed by ultrasonic relaxation of the singlet-quintet spin equilibrium of iron(II) complexes in solution. $J$. Am. Chem. Soc. 1978, 100, 3044-3050. 
(42) Baker, A.; Goodwin, H.; Rae, A. The crystal structure of Bis[2-(pyridin-2-ylamino)-4(pyridin-2-yl)thiazole]iron(II) Bis(tetrafluoroborate) trihydrate. Aust. J. Chem. 1984, $37,443-447$.

(43) Turner, J. W.; Schultz, F. A. Intramolecular and environmental contributions to electrode half-reaction entropies of $\mathrm{M}(\operatorname{tacn})_{2}^{3+/ 2+}(\mathrm{M}=\mathrm{Fe}, \mathrm{Co}, \mathrm{Ni}, \mathrm{Ru}$ and tacn $=1,4,7-$ triazacyclononae) redox couples. Inorg. Chem. 1999, 38, 358.

(44) Forbes, A.; Boeyens, J.; Hancock, R.; Wieghardt, K. Crystallographic Study of the LowSpin Iron(II) and Iron (III) Bis Complexes of 1,4,7-Triazacyclononane. Inorg. Chem. 1985, 24, 2926-2931.

(45) Chum, H. L.; Vanin, J. A.; Holanda, M. I. D. Tris(2-(aminomethyl)pyridine)iron(II): a new spin-state equilibrium in solution. Inorg. Chem. 1982, 21, 1146-1152.

(46) Chernyshov, D.; Hostettler, M.; Törnroos, K. W.; Bürgi, H.-B. Ordering Phenomena and Phase Transitions in a Spin-Crossover Compound-Uncovering the Nature of the Intermediate Phase of $\left[\mathrm{Fe}(2-\mathrm{pic})_{3}\right] \mathrm{Cl}_{2} \mathrm{EtOH}$. Angew. Chem. Int. Ed. 2003, 42, 38253830.

(47) Beattie, J. K.; Sutin, N.; Turner, D. H.; Flynn, G. W. Rate of intersystem crossing between $1 \mathrm{~A}$ and $5 \mathrm{Aa}$ states of an iron(II) complex in solutions. J. Am. Chem. Soc. 1973, 95, 2052-2054.

(48) Jesson, J. P.; Trofimenko, S.; Eaton, D. R. Spin equilibria in octahedral iron(II) poly((1pyrazolyl)-borates. J. Am. Chem. Soc. 1967, 89, 3158-3164.

(49) Oliver, J.; Hutchinson, B.; Mullica, D.; Milligan, W. Iron-Nitrogen Bond Lengths in Low-Spin and High-Spin Iron (II) Complexes with Poly (Pyrazolyl) Borate Ligands. Inorg. Chem. 1980, 19, 165-169. 
(50) Reeder, K. A.; Dose, E. V.; Wilson, L. J. Solution-state spin-equilibrium properties of the tris[2-(2-pyridyl)imidazole]iron(II) and tris[2-(2-pyridyl)benzimidazole]iron(II) cations. Inorg. Chem. 1978, 17, 1071-1075.

(51) Peng, S. M.; Chen, H. F. The crystal structures of metal complexes of 2-(2'pyridyl)benzimidazole and 2-(2'-pyridyl)benzimidazoate. Bull. Inst. Chem. Acad. Sin. 1990, 37, 49.

(52) Mcgarvey, J. J.; Lawthers, I.; Heremans, K.; Toftlund, H. Kinetics of spin-state interconversion in iron(II) complexes in solution as a function of pressure: activation volumes for the ${ }^{1} \mathrm{~A}_{1} \rightleftharpoons{ }^{5} \mathrm{~T}_{2}$ spin change. Inorg. Chem. 1990, 29, 252-256.

(53) McCusker, J.; Rheingold, A.; Hendrickson, D. Variable-Temperature Studies of LaserInitiated ${ }^{5} \mathrm{~T}_{2}$ to ${ }^{1} \mathrm{~A}_{1}$ Intersystem Crossing in Spin-Crossover Complexes: Empirical Correlations between Activation Parameters and Ligand Structure in a Series of Polypyridyl Ferrous Complexes. Inorg. Chem. 1996, 35, 2100-2112.

(54) Dolomanov, O. V.; Bourhis, L. J.; Gildea, R. J.; Howard, J. A. K.; Puschmann, H. OLEX2 : a complete structure solution, refinement and analysis program. J. Appl. Crystallogr. 2009, 42, 339-341.

(55) Kresse, G.; Hafner, J. Ab initio molecular dynamics for open-shell transition metals. Phys. Rev. B 1993, 48, 13115-13118.

(56) Kresse, G.; Hafner, J. Ab initio molecular-dynamics simulation of the liquid-metalamorphous-semiconductor transition in germanium. Phys. Rev. B 1994, 49, 1425114269.

(57) Kresse, G.; Furthmüller, J. Efficiency of ab-initio total energy calculations for metals and semiconductors using a plane-wave basis set. Comput. Mater. Sci. 1996, 6, 15-50. 
(58) Kresse, G.; Furthmüller, J. Efficient iterative schemes for ab initio total-energy calculations using a plane-wave basis set. Phys. Rev. B 1996, 54, 11169-11186.

(59) https://doi.org/10.14264/e619afa.

(60) Georges, A.; Medici, L. d.; Mravlje, J. Strong Correlations from Hund's Coupling. Annual Review of Condensed Matter Physics 2013, 4, 137-178. 
Graphical TOC Entry

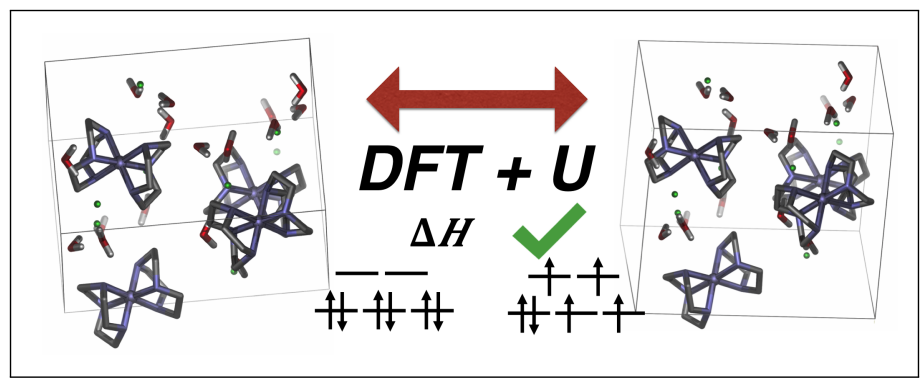

